\title{
Alopecia, CTCAE
}

National Cancer Institute

\section{Source}

National Cancer Institute. Alopecia, CT CAE. NCI Thesaurus. Code C57898.

A disorder characterized by a decrease in density of hair compared to normal for a given individual at a given age and body location. 